\title{
BMJ Open Discrimination in the workplace, reported by people with major depressive disorder: a cross-sectional study in 35 countries
}

\author{
E P M Brouwers, ${ }^{1}$ J Mathijssen, ${ }^{1}$ T Van Bortel, ${ }^{2}$ L Knifton, ${ }^{3} \mathrm{~K}$ Wahlbeck, ${ }^{4}$ \\ C Van Audenhove, ${ }^{5} \mathrm{~N}$ Kadri, ${ }^{6}$ Ch Chang, ${ }^{7}$ B R Goud, ${ }^{8}$ D Ballester, ${ }^{9}$ LF Tófoli, ${ }^{10}$ \\ $\mathrm{R}$ Bello, ${ }^{11} \mathrm{M}$ F Jorge-Monteiro, ${ }^{12} \mathrm{H}$ Zäske, ${ }^{13}$ I Milaćić, ${ }^{14} \mathrm{~A}$ Uçok, ${ }^{15} \mathrm{C}$ Bonetto, ${ }^{16}$ \\ A Lasalvia, ${ }^{16} \mathrm{G}$ Thornicroft, ${ }^{2} \mathrm{~J}$ Van Weeghel, ${ }^{1}$ the ASPEN/INDIGO Study Group*
}

To cite: Brouwers EPM, Mathijssen J, Van Bortel T, et al. Discrimination in the workplace, reported by people with major depressive disorder: a cross-sectional study in 35 countries. BMJ Open 2016;6:e009961. doi:10.1136/bmjopen-2015009961

- Prepublication history for this paper is available online. To view these files please visit the journal online (http://dx.doi.org/10.1136/ bmjopen-2015-009961).

Received 17 September 2015 Revised 16 November 2015 Accepted 2 December 2015

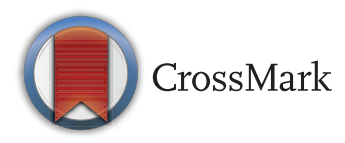

For numbered affiliations see end of article.

\section{Correspondence to} Dr EPM Brouwers; e.p.m.brouwers@ tilburguniversity.edu

\section{ABSTRACT}

Objective: Whereas employment has been shown to be beneficial for people with Major Depressive Disorder (MDD) across different cultures, employers' attitudes have been shown to be negative towards workers with MDD. This may form an important barrier to work participation. Today, little is known about how stigma and discrimination affect work participation of workers with MDD, especially from their own perspective. We aimed to assess, in a working age population including respondents with MDD from 35 countries: (1) if people with MDD anticipate and experience discrimination when trying to find or keep paid employment; (2) if participants in high, middle and lower developed countries differ in these respects; and (3) if discrimination experiences are related to actual employment status (ie, having a paid job or not). Method: Participants in this cross-sectional study $(\mathrm{N}=834)$ had a diagnosis of MDD in the previous 12 months. They were interviewed using the Discrimination and Stigma Scale (DISC-12). Analysis of variance and generalised linear mixed models were used to analyse the data.

Results: Overall, $62.5 \%$ had anticipated and/or experienced discrimination in the work setting. In very high developed countries, almost $60 \%$ of respondents had stopped themselves from applying for work, education or training because of anticipated discrimination. Having experienced workplace discrimination was independently related to unemployment.

Conclusions: Across different countries and cultures, people with MDD very frequently reported discrimination in the work setting. Effective interventions are needed to enhance work participation in people with MDD, focusing simultaneously on decreasing stigma in the work environment and on decreasing self-discrimination by empowering workers with MDD.

\section{INTRODUCTION}

Employment has many benefits that can contribute to the recovery of people with mental health problems. ${ }^{12}$ However, in many

\section{Strengths and limitations of this study}

Depression is a leading cause of disability worldwide, and for this study respondents with major depressive disorder (MDD) from as many as 35 countries were interviewed.

- This study examines the under-researched yet substantial problem of discrimination as a barrier to work participation of people with MDD.

- Interviews were used to gather direct self-reports rather than hypothetical scenarios or vignettes, which are often carried out in research on stigma and discrimination

- Limitations are the cross-sectional design of the study, and the fact that purposive sampling was used to recruit participants, which limits the generalisability of the results.

countries, participation and reintegration of people with mental health problems in the workforce is problematic. ${ }^{3}{ }^{4}$ Several factors cause this. Some are related to the individual, and some to the environment. An important barrier for full occupational participation and successful vocational integration is the stigma that is associated with mental health problems. ${ }^{5}$ Stigma is a mark or sign of disgrace usually eliciting negative attitudes to its bearer and can be seen as a problem associated with knowledge (ignorance), attitudes (prejudice) and behaviour (discrimination). ${ }^{6}$ Several studies have shown that although some cultural differences may exist, ${ }^{7}$ overall employers in many countries commonly express a range of concerns about hiring a potential employee with mental health problems. $^{8-10}$ Concerns reported include the belief that people with mental health problems have limited productivity and job performance, especially in tasks requiring cognitive skills, ${ }^{8} 11$ that they are unreliable and might pose threats to the safety of other 
employees, customers or themselves, ${ }^{11}$ or behave in a strange and unpredictable manner, and that there is potential for symptom relapse. ${ }^{8}$ In addition, the anticipation of discrimination by people with MDD may lead them not to apply for a job, in the expectation of failure or rejection.

Whereas most studies on mental health problems and discrimination in the workplace have focused on severe mental disorders such as schizophrenia, relatively few have focused on major depressive disorder (MDD). ${ }^{5}$ This is remarkable, as MDD is one of the leading causes of the global burden of disease. ${ }^{12}$ It is one of the most prevalent of all causes of disability ${ }^{13} 14$ and therefore an important public health problem. Across different countries and cultures, stigma and discrimination form an important barrier to work reintegration, although this topic has hardly been studied. In this context, the aim of this study was to assess: (1) if people with MDD of working age anticipate and experience discrimination because of their mental health problems when trying to find or keep paid employment; (2) if people with MDD of working age from high, middle and lower developed countries differ in this respect; and (3) if discrimination experiences when trying to find or keep paid employment are related to present work status (ie, having a paid job) in working aged people with MDD.

\section{METHODS}

\section{Study design}

Data were gathered as part of a larger study by the European Commission-funded Anti Stigma Programme European Network (ASPEN) study and the International Study of Discrimination and Stigma for Depression (INDIGO) research network. ${ }^{15}$ In a cross-sectional survey, people with a clinical diagnosis of major depressive disorder were interviewed in 35 countries. The ASPEN countries included Belgium, Bulgaria, England, Finland, France, Germany, Greece, Hungary, Italy, Lithuania, the Netherlands, Portugal, Romania, Scotland, Slovakia, Slovenia, Spain and Turkey. The countries participating through the INDIGO network included Australia, Brazil, Canada, Croatia, the Czech Republic, Egypt, India, Japan, Malaysia, Morocco, Nigeria, Pakistan, Serbia, Sri Lanka, Taiwan, Tunisia and Venezuela.

The design of this study was intentionally pragmatic so that as many as possible low income and middle income countries could participate using only locally available resources, because no external funding was available. Participants were recruited through local research staff who were asked to identify people attending specialist mental health services (either outpatient or day care in the public and private sectors) in the local area with a clinical diagnosis of MDD in the previous 12 months. All were asked to apply the Diagnostic and Statistical Manual Fourth Edition (DSM-IV) criteria in the same traditional way. Within centres, site directors were asked to identify a minimum of 25 participants who were, in their judgement, reasonably representative (as a group) of all people with a diagnosis of MDD attending specialist mental health services (either outpatient or day care in the public and private sectors in the local area). The minimum number of 25 for each site was defined for feasibility issues, particularly for non-European sites with no grant support. This method was intended to allow local staff to take into account the specific local service configuration and to draw participants from the whole range of appropriate local services. Staff at each site ensured that the sample had a spread across the adult age range (young people (18-25), working years (25-65), older adults $(\geq 65)$ ) and a clear representation of female participants as MDD is twice as prevalent in women as men. Response rates were unknown. Since the present study focused on the working age population, students $(\mathrm{N}=72)$ and retired respondents $(\mathrm{N}=168)$ were excluded from the analyses. Full details of the method have been published previously. ${ }^{15}$

\section{Procedure}

Data were gathered during face-to-face interviews in 2010, between 1 January and 31 December. Inclusion criteria were (1) a clinical diagnosis of major depressive disorder during the previous 12 months (single episode or recurrent), as based on the DSM-IV criteria; (2) ability to speak and understand the main local language; and (3) aged 18 years or older. Individuals who were receiving psychiatric inpatient care during recruitment were excluded. The study was approved by the appropriate ethical review board at each study site. After complete description of the study to the subjects, written informed consent was obtained.

\section{Measures}

Participants were assessed face to face by independent researchers not involved in the care process using the standardised Discrimination and Stigma scale (V.12), a structured interview for recording the discrimination experienced by an individual with a mental health problem. ${ }^{16} 17$ The DISC-12 interview starts with the statement 'Discrimination and stigma occur when people are treated unfairly because they are seen as being different from others. This interview asks about how participants have been affected by discrimination and stigma because of mental health problems'. The instrument consists of 32 questions, assessing discrimination in several life domains, such as marriage, parenting, housing and leisure. For the present paper, only the items that referred to discrimination in the work environment are reported on. For anticipated discrimination, the items used in this study were: 'Because of how others might respond to your mental health problem, have you stopped yourself from applying for work?' and 'Because of how others might respond to your mental health problem, have you stopped yourself from applying for education and training?' For experienced 
discrimination, the items used were 'Because of how others might respond to your mental health problem, have you been treated unfairly in finding a job?' and 'Because of how others might respond to your mental health problem, have you been treated unfairly in keeping a job?' All questions were answered on a fourpoint Likert scale $(0=$ not at all, $1=\mathrm{a}$ little, $2=$ moderately and $3=\mathrm{a}$ lot).

For the second research question, consistent with the methodology of a previous ASPEN/INDIGO paper, ${ }^{18}$ countries were divided into groups according to the Human Development Index (HDI). The HDI is a summary measure of human development established by the United Nations, ${ }^{19}$ which measures the average achievements of a country in three basic dimensions of human development: (1) long and healthy life (operationalised as life expectancy at birth), (2) access to knowledge, (ie, the mean number of years of schooling) and (3) standard of living, (ie, gross national income per capita). Since data were gathered in 2010, the HDI statistic of that year was used. Countries with a very high HDI score were England, Australia, Finland, Germany, Canada, Italy, Portugal, Belgium, France, Japan, Greece, the Netherlands, Scotland, Slovakia, Slovenia, Spain, the Czech Republic, Taiwan and Hungary. Countries with a high HDI score were Turkey, Malaysia, Brazil, Serbia, Bulgaria, Venezuela, Tunisia, Lithuania, Romania and Croatia. Since few countries had a low HDI, the medium low and low HDI group were taken together as one group for the analyses. This medium/low HDI group included Egypt, India, Morocco, Nigeria, Pakistan and Sri Lanka.

Internalised stigma, one of the independent variables included in the analyses for the third research question, was measured with the Internalized Stigma of Mental Illness Scale (ISMI) ${ }^{20}$ Internalised stigma refers to the inner subjective experience of stigma and its psychological effects resulting from applying negative stereotypes and stigmatising attitudes to oneself. The ISMI is a 29-item instrument for self-rated assessment of the subjective experience of stigma, with higher scores indicating higher internalised stigma. Here, the total score on the ISMI was used.

\section{Statistical analyses}

All analyses were performed using SPSS V.19. All p values were two-tailed with an accepted significance level of 0.05 . For the first research question, percentages of anticipated and experienced workplace discrimination were reported per country. For the second research question, two separate ANOVAS were conducted, the first of which was with anticipated workplace discrimination as the dependent variable and HDI level as the independent variable. A second ANOVA analysis was conducted with experienced workplace discrimination as the dependent variable and HDI level as the independent variable. For the first and second research questions, answers to the questions on anticipated and experienced workplace discrimination were dichotomised into 'No' ('not at all') and 'Yes' ('a little', 'moderately', 'a lot'). For the third research question, multivariate logistic regression analysis was performed, using work status as the dependent variable (defined as $0=$ no paid employment and $1=$ employed), and 10 independent variables, including experienced workplace discrimination. First, univariate analyses were conducted including the following independent variables that were expected to be related to job outcome: experienced workplace discrimination, gender, age, ethnicity (ie, belonging to an ethnic minority), level of education, marital status, previous psychiatric treatment, age of first contact with mental health services, internalised stigma (ISMI total score) and HDI. Second, all variables that showed a significant relationship with the dependent variable on a univariate level $(p<0.05)$ were included in the multivariable logistic regression analysis.

\section{RESULTS}

A total of 834 people with MDD across 35 different countries were individually interviewed for this study. About half of all participants were married or cohabiting, and two-thirds of the participants were women. Characteristics of the sample are shown in table 1 . Although there were differences in employment rate across sites, the employment rates per HDI group did not differ significantly.

As shown in table 2, for each separate question, about $40-50 \%$ of the participants indicated that discrimination was not a problem for them. However, when looking at the four items combined, about two-thirds $(62.5 \%)$ of the total sample reported anticipated and/or experienced discrimination in the work setting due to their mental health problem. Almost one-third of participants indicated to have stopped themselves from applying for work because of anticipated discrimination.

Regarding the second research question, significant differences were found between the groups with different HDI levels. Specifically, participants in countries with a very high HDI reported significantly more often anticipated $\left(\chi^{2}=26.01 \quad(\mathrm{df}=2), \mathrm{p}<0.01\right)$ and also experienced $\left(\chi^{2}=7.25 \quad(\mathrm{df}=2), \mathrm{p}<0.05\right)$ discrimination than participants in countries with moderate/low HDI (see figure 1). As can also be seen from figure 1, in all three groups the anticipated workplace discrimination scores were higher than the experienced workplace discrimination scores.

Concerning the third research question, as can be seen in table 3, several variables were not related to work status on a univariate level (ie, 'belonging to an ethnic minority', 'marital status', 'age of first contact with mental health services' and 'HDI'), for which reason they were not included in the multivariable model. Results from the multilevel logistic regression analysis showed that experienced workplace discrimination was independently and positively related to 
Table 1 Characteristics of the sample $(\mathrm{N}=834)$

\begin{tabular}{|c|c|}
\hline \multicolumn{2}{|l|}{ Demographic characteristics } \\
\hline Age (mean, SD) & $42.7(11.9)$ \\
\hline Female gender (\%) & 66.9 \\
\hline \multicolumn{2}{|l|}{ Education (\%) } \\
\hline $\begin{array}{l}\text { None, primary (age } \leq 12) \text {, secondary } \\
\text { ( } \leq 15-16 \text { years) or vocational qualification }\end{array}$ & 43.8 \\
\hline $\begin{array}{l}\text { Diploma, degree or postgraduate } \\
\text { qualification }\end{array}$ & 56.2 \\
\hline \multicolumn{2}{|l|}{ Marital status (\%) } \\
\hline Married or cohabiting & 52.2 \\
\hline Single or non-cohabiting partner & 25.9 \\
\hline Widowed, separated, divorced & 21.7 \\
\hline Belongs to ethnic minority (\%) & 8.2 \\
\hline \multicolumn{2}{|l|}{ Human Development Index score* } \\
\hline Very high HDI countries & 47.0 \\
\hline High HDI countries & 28.2 \\
\hline Medium HDI countries & 14.0 \\
\hline Low HDI countries & 10.8 \\
\hline \multicolumn{2}{|l|}{ Mental health characteristics } \\
\hline Ever admitted for psychiatric care (\%) & 36 \\
\hline $\begin{array}{l}\text { Age of first contact with mental health } \\
\text { services (mean, SD) }\end{array}$ & $33.6(11.8)$ \\
\hline Internalised stigma total score† (mean, SD) & $2.4(0.55)$ \\
\hline \multicolumn{2}{|l|}{ Work-related characteristics } \\
\hline \multicolumn{2}{|l|}{ Employment } \\
\hline Full-time or part-time & 51.2 \\
\hline $\begin{array}{l}\text { Volunteer, or working in a sheltered } \\
\text { accommodation or at home }\end{array}$ & 13.1 \\
\hline Looking for a job & 14.4 \\
\hline Unemployed, not looking for a jobł & 21.3 \\
\hline \multicolumn{2}{|c|}{$\begin{array}{l}\text { *HDI, United Nations Development Programme. }{ }^{19} \\
\text { †Total score on the Internalised Stigma of Mental Illness scale. } \\
\text { Scale ranges from } 1 \text { to } 4, \text { with higher scores indicating higher } \\
\text { internalised stigma. } \\
\text { 'Combination of 'Would like to work but afraid to loose benefits', } \\
\text { 'unable to work', 'choose not to work'. }\end{array}$} \\
\hline
\end{tabular}

unemployment $(0.61,95 \%$ CI 0.43 to 0.86$)$. Other variables that were significantly related to unemployment were 'low educational level' $(0.48,95 \%$ CI 0.34 to 0.69$)$ and 'having ever been admitted to psychiatric treatment' $(0.55,95 \%$ CI 0.38 to 0.79$)$.

\section{DISCUSSION}

The results of this study show that as many as $62.5 \%$ of participants reported having anticipated and/or experienced discrimination in the work setting. Anticipated workplace discrimination was reported more often than experienced workplace discrimination. Participants from countries with a very high HDI reported significantly more often anticipated and experienced workplace discrimination, although even in the medium/low HDI group about one-third of participants reported discrimination in the work setting. Regarding the third research question, it was found that experienced workplace discrimination was indeed independently related to unemployment.

These findings show that discrimination in the workplace is a common problem in many countries
Table 2 Responses to the DISC- $12^{*}$ questions related to employment $(\mathrm{N}=834)$

\begin{tabular}{|c|c|}
\hline & $\mathrm{N}(\%)$ \\
\hline \multicolumn{2}{|c|}{ Anticipated discrimination } \\
\hline \multicolumn{2}{|c|}{...have you stopped yourself from applying for work? } \\
\hline not at all & $338(40.5)$ \\
\hline a little & $63(7.6)$ \\
\hline moderately & $65(7.8)$ \\
\hline a lot & $109(13.1)$ \\
\hline not applicable & $239(28.7)$ \\
\hline \multicolumn{2}{|c|}{$\begin{array}{l}\text {...have you stopped yourself from applying for education o } \\
\text { training courses? }\end{array}$} \\
\hline not at all & $373(44.7)$ \\
\hline a little & $72(8.6)$ \\
\hline moderately & $39(4.7)$ \\
\hline a lot & $67(8.0)$ \\
\hline not applicable & $262(31.4)$ \\
\hline \multicolumn{2}{|c|}{ Experienced discrimination } \\
\hline \multicolumn{2}{|c|}{...have you been treated unfairly in finding a job? } \\
\hline not at all & $402(48.2)$ \\
\hline a little & $41(4.9)$ \\
\hline moderately & $35(4.2)$ \\
\hline a lot & $45(5.4)$ \\
\hline not applicable & $307(36.8)$ \\
\hline \multicolumn{2}{|c|}{...have you been treated unfairly in keeping a job? } \\
\hline not at all & $423(50.7)$ \\
\hline a little & $61(7.3)$ \\
\hline moderately & $57(6.8)$ \\
\hline a lot & 77 (9.2) \\
\hline not applicable & $213(25.5)$ \\
\hline
\end{tabular}

worldwide. Considering that inpatients were excluded from the study, for the total group of people with MDD these percentages may even be much higher. These findings are consistent with those of a recent review ${ }^{21}$ and

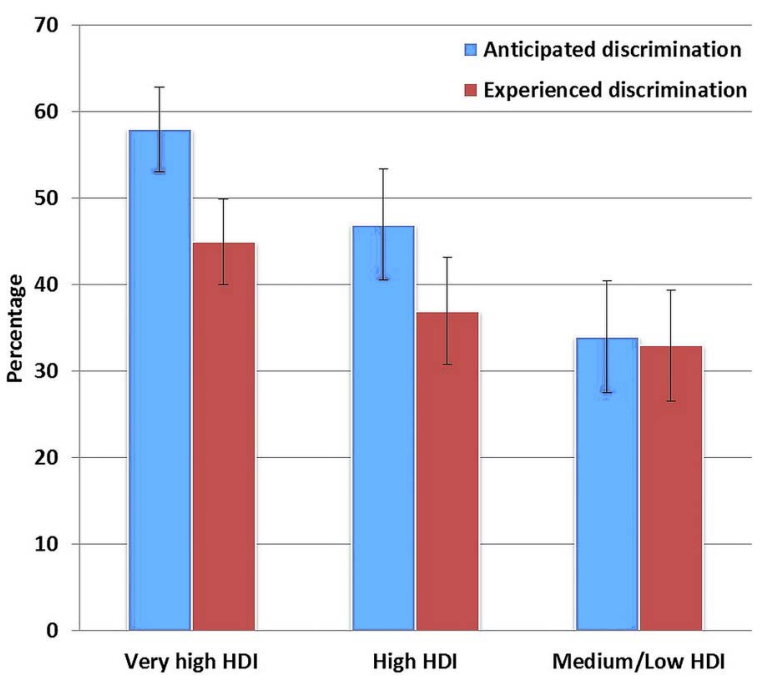

Figure 1 Percentages and 95\% Cls of respondents who reported having anticipated and experienced discrimination in the work setting in very high, high, moderately and lower developed countries. HDI, Human Development Index. 
Table 3 Multivariable logistic regression analysis work status

\section{Univariable models \\ ORs $(95 \% \mathrm{Cl})$}

Multivariable model ORs $(95 \% \mathrm{Cl})$

\begin{tabular}{|c|c|c|}
\hline \multicolumn{3}{|l|}{ Experienced discrimination } \\
\hline No & Ref & Ref \\
\hline Yes & $0.63(0.45 \text { to } 0.88)^{\star *}$ & $0.61(0.43 \text { to } 0.86)^{\star \star}$ \\
\hline \multicolumn{3}{|l|}{ Sex } \\
\hline Male & Ref & Ref \\
\hline Female & $0.68(0.50 \text { to } 0.92)^{*}$ & $0.79(0.55$ to 1.14$)$ \\
\hline Age & $0.99(0.97 \text { to } 1.00)^{*}$ & 0.99 (0.98 to 1.01$)$ \\
\hline Belongs to ethnic minority & & - \\
\hline No & Ref & \\
\hline Yes & $0.88(0.50$ to 1.55$)$ & \\
\hline \multicolumn{3}{|l|}{ Education } \\
\hline Diploma, degree or postgraduate qualification & Ref & Ref \\
\hline $\begin{array}{l}\text { None, primary (age } \leq 12) \text {, secondary } \\
\text { ( } \leq 15-16 \text { years) or vocational qualification }\end{array}$ & $0.44(0.33 \text { to } 0.59)^{\star *}$ & $0.48(0.34 \text { to } 0.69)^{\star *}$ \\
\hline \multicolumn{3}{|l|}{ Marital status } \\
\hline Married or cohabiting & Ref & - \\
\hline Single or non-cohabiting partner & $0.72(0.50$ to 1.03$)$ & \\
\hline Widowed, separated or divorced & $0.86(0.61$ to 1.21$)$ & \\
\hline \multicolumn{3}{|l|}{ Ever admitted for psychiatric treatment } \\
\hline No & Ref & Ref \\
\hline Yes & $0.61(0.45 \text { to } 0.84)^{\star \star}$ & $0.55(0.38 \text { to } 0.79)^{\star \star}$ \\
\hline Age of first contact with mental health services & $1.00(0.99$ to 1.01$)$ & - \\
\hline ISMI total & $0.66(0.50 \text { to } 0.86)^{* *}$ & $0.72(0.52$ to 1.00$)$ \\
\hline \multicolumn{3}{|l|}{ HDI } \\
\hline Low/medium HDI countries & Ref & - \\
\hline High HDI countries & $1.43(0.71$ to 2.85$)$ & \\
\hline Very High HDI countries & $1.34(0.71$ to 2.50$)$ & \\
\hline
\end{tabular}

that of a large Australian study on the experiences and perspectives of people with MDD. ${ }^{22}$ Here, participants indicated that stigma was a considerable problem, particularly regarding employment. In a similar German study, $81.5 \%$ of the 55 participants who had experienced a depressive episode anticipated stigmatisation in the occupational setting. ${ }^{23}$ These studies from the depressed individual's perspective are in line with results of studies from employers' perspectives. Such studies have shown that employers tend to have negative attitudes towards people with mental health problems. ${ }^{5-7}$

An important finding of this study was that participants anticipated workplace discrimination more often than they had actually experienced it. In another study, Uçok $e t a l^{8}$ found that anticipated discrimination was not necessarily associated with experienced discrimination. Similar to our results, Angermeyer et $a l^{23}$ also found anticipated discrimination to be higher than experienced discrimination, and suggested that it could result in a tendency to avoid situations with a high risk of stigma. Corrigan et $a l^{24}$ described this 'why try' effect as an overarching phenomenon encompassing self-stigma, followed by low self-esteem and self-efficacy, and a diminished behaviour to pursue life goals. However, not only people with mental ill health themselves anticipate to be discriminated in the workplace. A recent population-based survey of working adults in Canada showed that a third of workers would not tell their managers if they experienced mental health problems, mostly out of fear of damaging their careers. ${ }^{25}$ Hence, findings from these studies and this study underline the clear need for interventions focusing on the empowerment of people with MDD in the work environment. Peer support plays an important role in enhancing empowerment and decreasing self-stigma ${ }^{20}$ and may be useful in such programmes.

Since mental health problems are highly prevalent, ${ }^{13} 26$ but people with these disorders are often reluctant to disclose their condition, ${ }^{21} 222728$ employers usually are not aware of the fact that many of their employees have mental health problems. Although this is a major impediment for work adaptations, authors of a recent vignette study concluded that concealment of mental health problems may actually be wise, as employers tended to think more negatively about a worker with depression than with a physical disorder under the exact 
same circumstances. ${ }^{29}$ Recently, several studies have been conducted on the topic of disclosure of mental illness in the workplace..$^{21} 2728{ }^{30}$ For instance, a decision aid for employees on whether or not to disclose their mental health problems to an employer has been developed, ${ }^{31} 32$ which has been shown to effectively reduce decisional conflict in employees with mental health problems. ${ }^{31}$ The findings of the present and other studies ${ }^{21} 25$ suggest that future programmes aimed at reducing stigma and discrimination should also involve stakeholders from the environment such as employers and occupational health professionals as they play a major role in, for instance, whether or not temporary workplace reasonable adjustments or accommodations are made. Boot $e t a \hat{l}^{33}$ showed that workplace adjustments are associated with a reduction in sick leave duration and that $43 \%$ of workers with mental health problems reported a need for work adjustments.

The results of this study indicated that in very highly developed countries, significantly higher percentages of workplace discrimination were reported as compared to countries with a low/medium developmental score (research question 2). These findings differ from those of an intercultural study on employers' attitudes towards hiring and accommodating a person with disabilities at work. ${ }^{10}$ Here, it was found that Chinese employers were less likely to endorse hiring people with psychiatric disabilities than employers from the USA or Hong Kong. However, it should be noticed that within one HDI group, many different countries and cultures are represented which limits generalisability.

Whereas the size of this study, including 35 countries, is a considerable strength, the number of people interviewed per country was too small to draw any conclusions at country level. Nevertheless, the results indicated that even in countries with a medium to low developmental score, about one-third of participants reported discrimination in the work setting. Future research should focus on differences between countries and study, for instance, the effects of legislation. However, legislation will not entirely solve the problem, as legislation does not address self-stigma and also in countries with more advanced equality legislation experienced workplace discrimination rates were still high.

We also found that experienced workplace discrimination was significantly related to unemployment (research question 3). These findings are similar to those of a large household interview survey in six European countries. Specifically, they found that in participants with a mental health problem, perceived stigma was significantly associated with being unemployed, as well as with a decreased quality of life, higher work and role limitations and higher social limitations. ${ }^{34}$ An explanation for the finding that experienced workplace discrimination was independently related to unemployment is that the social stigma attached to mental health problems among employers may hinder them from hiring an employee with MDD. ${ }^{11} 29$ Alternatively this finding may be explained by the fact that during job interviews, applicants with MDD may not get the position because MDD is characterised by a variety of symptoms that may be disadvantageous during job interviews, such as markedly diminished interest in activities, impaired ability to think, concentrate or make decisions, fatigue, increased irritability and low self-worth. ${ }^{20}$ These symptoms may influence both applicants' verbal and non-verbal behaviour, thereby diminishing their chances of being appointed.

When considering the results of this study, several limitations need to be taken into account. First, apart from the four items on the DISC questionnaire that measured anticipated and experienced workplace discrimination, little additional information was available on how participants perceived their work setting and why they felt discriminated. Future qualitative and longitudinal studies are needed to address this in more detail, focusing on the role of stakeholders such as supervisors, employers, colleagues and occupational health professionals. A second limitation is that the design of the study was crosssectional, for which reason no causality can be assumed. Hence, workplace discrimination may lead to unemployment, but unemployment may also lead to feelings of being discriminated against. Third, purposive sampling was used to recruit participants. This limits the generalisability of the results, as participants do not necessarily represent true prevalent cases in the community.

In conclusion, the results suggest that anticipated and experienced discrimination in the workplace is a highly common phenomenon in higher as well as in lower developed countries across the world. The topic of overcoming stigma and discrimination has been underresearched so far ${ }^{35}$ but may offer new ways to improve work participation of people with MDD. In many countries, mental health problems such as MDD are associated with high costs for society, due to unemployment, absences and at-work performance deficits. ${ }^{36-38}$ Previous studies have called for research addressing workplace environment issues to improve work participation of people with MDD. ${ }^{36}{ }^{38}$ Stigma and workplace discrimination are such issues and there is a clear need for effective interventions.

\section{Author affiliations}

${ }^{1}$ Department of Tranzo, Tilburg University, Tilburg, The Netherlands

${ }^{2}$ King's College London, Institute of Psychiatry, London, UK

${ }^{3}$ Mental Health Foundation, Glasgow, UK

${ }^{4}$ National Institute for Health and Welfare, Vaasa, Finland

${ }^{5}$ Katholieke Universiteit Leuven, Leuven, Belgium

${ }^{6} \mathrm{lbn}$ Rushd University Psychiatric Centre, Casablanca, Morocco

${ }^{7}$ Department of Psychiatry, Chi Mei Medical Centre, Tainan, Taiwan

${ }^{8}$ St John's Medical College Hospital, St John's National Academy of Health

Sciences, Bangalore, India

${ }^{9}$ Sistema de Saúde Mãe de Deus, Porto Alegre, Brazil

${ }^{10}$ Universidade Federal do Ceara, Campus Sobral, Brazil

${ }^{11}$ Hospital Universitario de Caracas, Caracas, Venezuela

${ }^{12}$ Associacao para o Estudo e Integracao Psicossocial, Lisbon, Portugal

${ }^{13}$ Heinrich-Heine Universitat Dusseldorf, Rheinische Kliniken Dusseldorf, Germany 
${ }^{14}$ Faculty for Special Education and Rehabilitation, Belgrade, Serbia ${ }^{15}$ Foundation of Psychiatry Clinic of Medical Faculty of Istanbul, Istanbul, Turkey

${ }^{16}$ Department of Public Health and Community Medicine, Section of Psychiatry, University of Verona, Verona, Italy

Acknowledgements The ASPEN/INDIGO* staff at coordinating centres: Graham Thornicroft, Tine Van Bortel, Samantha Treacy, Elaine Brohan, Shuntaro Ando, Diana Rose (King's College London, Institute of Psychiatry, London, England); Kristian Wahlbeck, Esa Aromaa, Johanna Nordmyr, Fredrica Nyqvist, Carolina Herberts (National Institute for Health and Welfare, Vaasa, Finland); Oliver Lewis, Jasna Russo, Dorottya Karsay, Rea Maglajlic (Mental Disability Advocacy Centre, Budapest, Hungary); Antonio Lasalvia, Silvia Zoppei, Doriana Cristofalo, Chiara Bonetto (Department of Public Health and Community Medicine, Section of Psychiatry, University of Verona, Italy); Isabella Goldie, Lee Knifton, Neil Quinn (Mental Health Foundation, Glasgow, Scotland); Norman Sartorius (Association for the improvement of mental health programmes (AMH), Geneva, Switzerland). The ASPEN/INDIGO staff at partner centres: Chantal Van Audenhove, Gert Scheerder, Else Tambuyzer (Katholieke Universiteit Leuven, Belgium); Valentina Hristakeva, Dimitar Germanov (Global Initiative on Psychiatry Sofia, Bulgaria); Jean Luc Roelandt, Simon Vasseur Bacle, Nicolas Daumerie, Aude Caria (Etablissement Public Santé Mentale Lille-Métropole (EPSM/CCOMS), France); Harald Zaske, Wolfgang Gaebel (Heinrich-Heine Universitat Dusseldorf, Rheinische Kliniken Dusseldorf, Germany); Marina Economou, Eleni Louki, Lily Peppou, Klio Geroulanou (University Mental Health Institute (UMHRI (EPIPSI), Greece); Judit Harangozo, Julia Sebes, Gabor Csukly (Awakenings Foundation, Hungary); Giuseppe Rossi, Mariangela Lanfredi, Laura Pedrini (IRCCS Istituto Centro San Giovanni di Dio Fatebenefratelli, Brescia, Italy; Arunas Germanavicius, Natalja Markovskaja, Vytis Valantinas (Vilnius University, Lithuania); Jaap van Weeghel, Jenny Boumans, Eleonoor Willemsen, Annette Plooy (Stichting Kenniscentrum Phrenos (KcP), The Netherlands); Teresa Duarte, Fatima Jorge Monteiro (Associacao para o Estudo e Integracao Psicossocial, Portugal); Radu Teodorescu, luliana Radu, Elena Pana (Asociatia din Romania de Psihiatrie Comunitara, Romania; Janka Hurova, Dita Leczova (Association for Mental Health INTEGRA, o. z., Slovakia); Vesna Svab, Nina Konecnik (University Psychiatric Hospital, Slovenia); Blanca Reneses, Juan J Lopez-Ibor, Nerea Palomares, Camila Bayon (Instituto de Psiquiatria at the Hospital Universitario San Carlos, Spain); Alp Uçok, Gulsah Karaday (Foundation of Psychiatry Clinic of Medical Faculty of Istanbul (PAP), Turkey); Nicholas Glozier, Nicole Cockayne (Brain \& Mind Research Institute, Sydney Medical School, University of Sydney, Australia); Luís Fernando Tófoli, Maria Suely Alves Costa (Universidade Federal do Ceará, Campus Sobral, Brazil); Roumen Milev, Teresa Garrah, Liane Tackaberry, Heather Stuart (Department of Psychiatry, Queen's University, Canada/Providence Care, Mental Health Services, Kingston, Ontario, Canada; Branka Aukst Margetic, Petra Folnegovic Groiæ (Department of Psychiatry, University Hospital Centre ZagrebMiro Jakovljeviæ, Croatia); Barbora Wenigová, elepová Pavla (Centre for Mental Health Care Development, Prague, Czech Republic); Doaa Nader Radwan (Institute of Psychiatry, Ain Shams University, Cairo, Egypt); Pradeep Johnson, Ramakrishna Goud, Nandesh, Geetha Jayaram (St. John's Medical College Hospital, St John's National Academy of Health Sciences, Bangalore, India; Shuntaro Ando (Social Psychiatry, Tokyo Metropolitan Institute of Medical Science, Tokyo, Japan; Yuriko Suzuki, Tsuyoshi Akiyama, Asami Matsunaga, Peter Bernick (NTT Kanto Hospital, Japan); Bawo James (Federal Neuropsychiatric Hospital, USELU, Benin City, Nigeria; Bolanle Ola, Olugbenga Owoeye (Federal Neuropsychiatric Hospital Yaba, Lagos, Nigeria); Yewande Oshodi (Department of Psychiatry, College of Medicine University of Lagos and Lagos University Teaching Hospital, Lagos, Nigeria; Jibril Abdulmalik (Federal Neuropsychiatric Hospital, Maiduguri, Nigeria); Kok-Yoon Chee, Norhayati Ali (Kuala Lumpur Hospital and Selayang Hospital, Malaysia); Nadia Kadri, Dounia Belghazi, Yassine Anwar (Ibn Rushd University Psychiatric Centre, Casablanca, Morocco); Nashi Khan, Rukhsana Kausar (University of the Punjab, Department of Applied Psychology and Centre for Clinical Psychology, Lahore, Pakistan); Ivona Milacic Vidojevic (Faculty for Special Education and Rehabilitation, Belgrade, Serbia); Athula Sumathipala (Institute of Psychiatry, King's College London/Institute for Research and Development, Sri Lanka); Chih-Cheng Chang (Chi Mei Medical Centre, Department of Psychiatry, Tainan), Taiwan; Fethi Nacef, Uta Ouali, Hayet Ouertani, Rabaa
Jomli, Abdelhafidh Ouertani, Khadija Kaaniche (Razi Hospital Manouba, Department of Psychiatry, Tunis, Tunisia); Ricardo Bello, Manuel Ortega, Arturo Melone, María Andréina, Francisco Marco, Arturo Ríos, Ernesto Rodríguez, Arianna Laguado (Hospital Universitario de Caracas, Caracas, Venezuela). GT is supported by the National Institute for Health Research (NIHR) Collaboration for Leadership in Applied Health Research and Care South London at King's College London Foundation Trust. The views expressed are those of the author(s) and not necessarily those of the NHS, the NIHR or the Department of Health. GT acknowledges financial support from the Department of Health via the National Institute for Health Research (NIHR) Biomedical Research Centre and Dementia Unit awarded to South London and Maudsley NHS Foundation Trust in partnership with King's College London and King's College Hospital NHS Foundation Trust. GT is supported by the European Union Seventh Framework Programme (FP7/ 2007-2013) Emerald project.

Contributors The original study design and protocol were written by AL, TVB and GT. AL, TVB, CB, KW, CVA, JVW, IM and GT coordinated data gathering in the participating sites. EPMB, JVW, JM AL and CB participated in the data analysis and interpretation. The report was written by EPMB, JVW, JM, TVB and GT and was edited by all authors, who also approved of the final version.

Funding This report arises from the project Anti Stigma Programme European Network (ASPEN) which has received funding from the European Union in the framework of the Public Health Programme.

\section{Competing interests None declared.}

Patient consent Obtained.

Ethics approval The study was approved by the appropriate ethical review board at each study site.

Provenance and peer review Not commissioned; externally peer reviewed.

Data sharing statement No additional data are available.

Open Access This is an Open Access article distributed in accordance with the Creative Commons Attribution Non Commercial (CC BY-NC 4.0) license, which permits others to distribute, remix, adapt, build upon this work noncommercially, and license their derivative works on different terms, provided the original work is properly cited and the use is non-commercial. See: http:// creativecommons.org/licenses/by-nc/4.0/

\section{REFERENCES}

1. Eklund M, Hansson L, Ahlqvist C. The importance of work as compared to other forms of daily occupations for wellbeing and functioning among persons with long-term mental illness. Community Ment HIt J 2004;40:465-77.

2. Van der Noordt M, IJzelenberg $H$, Droomers $M$, et al. Health effects of employment: a systematic review of prospective studies. Occup Environ Med 2014;71:730-6.

3. Gilmour H, Patten SB. Depression and work impairment. Health Rep 2007;18:9-22

4. Henderson M, Madan I, Hotopf M. Work and mental health in the UK. BMJ 2014;348:g2256.

5. Angermeyer MC. Important to investigate the dynamics of the stigma process. Healthc Pap 2004;5:112-13.

6. Thornicroft G, Rose D, Kassam A, et al. Stigma: ignorance, prejudice or discrimination? Br J Psychiatry 2007;190:192-3.

7. Tsang HW, Angell B, Corrigan PW, et al. A cross-cultural study of employers' concerns about hiring people with psychotic disorders: implications for recovery. Soc Psych Psych Epid 2007;42:723-33.

8. Uçok A, Brohan E, Rose D, et al. Anticipated discrimination among people with schizophrenia. Acta Psychiat Scand 2012;125:77-83.

9. Mangili E, Ponteri M, Buizza C, et al. Attitudes toward disabilitues and mental illness in work settings: a review. Epidemiol Psichiatr Soc 2004;13:29-46.

10. Corrigan PW, Kuwabara S, Tsang HW, et al. Disability and work-related attitudes in employers from Beijing, Chicago and Hong Kong. Int J Rehabil Res 2008;31:347-50.

11. Scheid TL. Stigma as a barrier to employment: mental disability and the Americans with Disabilities Act. Int $J$ Law Psychiat 2005;28:670-90.

12. Moussavi S, Chatterij S, Verdes E, et al. Depression, chronic diseases, and decrements in health: results from the World Health Surveys. Lancet 2007;370:851-8. 
13. Kessler RC, Akiskal HS, Ames M, et al. Prevalence and effects of mood disorders on work performance in a nationally representative sample of U.S. workers. Am J Psychiatry 2006;163:1561-8.

14. Lopez A, Mathers C, Ezzati M, et al. Global and regional burden of disease and risk factors, 2001: systematic analyses of population health data. Lancet 2006;367:1747-57.

15. Lasalvia A, Zoppei S, Van Bortel T, et al. Global pattern of experienced and anticipated discrimination reported by people with depressive disorder: a cross-sectional survey. Lancet 2013;381:55-62.

16. Thornicroft G, Brohan E, Rose D, et al. Global pattern of anticipated and experienced discrimination against people with schizophrenia: a cross-sectional survey. Lancet 2009;373:408-15.

17. Brohan E, Clement S, Rose D, et al. Development and psychometric evaluation of the Discrimination and Stigma Scale (DISC). Psychiat Res 2013;208:33-40.

18. Lasalvia A, Van Bortel $\mathrm{T}$, Bonetto $\mathrm{C}$, et al. Cross-national variations in reported discrimination among people with major depression worldwide: the ASPEN/INDIGO international study. Br J Psychiatry 2015;207:507-14.

19. United Nations Development Programme (UNDP). Human Development Report 2010. The Wealth of Nations: Pathways to Human Development. UNDP, 2010.

20. Boyd Ritsher J, Otilingam PG, Grajales M. Internalized stigma of mental illness: psychometric properties of a new measure. Psychiat Res 2003;121:31-49.

21. Brohan $\mathrm{E}$, Henderson $\mathrm{C}$, Wheat $\mathrm{K}$, et al. Systematic review of beliefs, behaviours and influencing factors associated with disclosure of a mental health problem in the workplace. BMC Psychiatr 2012;12:11.

22. McNair B, Highet N, Hickie I, et al. Exploring the perspectives of people whose lives have been affected by depression. Med J Aust 2002;176(Suppl):S69-76.

23. Angermeyer MC, Beck M, Dietrich S, et al. The stigma of mental illness: patients' anticipations and experiences. Int J Soc Psychiatr 2004:50:153-62.

24. Corrigan PW, Larson J, Ruesch N. Self-Stigma and the "why-try" effect: impact on life goals and evidence-based practices. World Psychiatry 2009;8:75-81.

25. Dewa C. Worker attitudes towards mental health problems and disclosure. J Occup Environ Med 2014;5:175-86.
26. De Graaf R, Ten Have M, Van Gool C, et al. Prevalence of mental disorders and trends from 1996 to 2009. Results from the Netherlands Mental Health Survey and Incidence Study-2. Soc Psych Psych Epid 2012;47:203-13.

27. Toth KE, Dewa CS. Employee decision-making about disclosure of a mental disorder at work. J Occup Rehabil 2014;24:732-46.

28. Moll SE. The web of silence: a qualitative case study of early intervention and support for healthcare workers with mental ill-health. BMC Public Health 2014;14:138.

29. Mendel R, Kissling W, Reichhart T, et al. Managers' reactions towards employees' disclosure of psychiatric or somatic diagnoses. Epidemiol Psychiatr Sci 2015;24:146-9.

30. Brohan E, Evans-Lacko S, Henderson C, et al. Disclosure of a mental health problem in the employment context: qualitative study of beliefs and experiences. Epidem Psychiatr Sci 2014;23:289-300.

31. Henderson C, Brohan E, Clement S, et al. Decision aid on disclosure of mental health status to an employer: feasibility and outcomes of a randomised controlled trial. Br J Psychiatry 2013;203:350-7.

32. Henderson $\mathrm{C}$, Brohan $\mathrm{E}$, Clement S, et al. A decision aid to assist decisions on disclosure of mental health status to an employer: protocol for the CORAL exploratory randomised controlled trial. BMC Psychiatry 2012;12:133.

33. Boot CR, Van den Heuvel SG, Bültmann U, et al. Work adjustments in a representative sample of employees with a chronic disease in the Netherlands. J Occup Rehabil 2013;23:200-8.

34. Alonso J, Buron A, Rojas-Ferreras S, et al. Perceived stigma among individuals with common mental disorders. $J$ Affect Disord 2009;118:180-6.

35. Evans-Lacko S, Courtin E, Fiorillo A, et al. The state of the art in European research on reducing social exclusion and stigma related to mental health: a systematic mapping of the literature. Eur Psychiatry 2014;29:381-9.

36. Lerner D, Henke R. What does research tell us about depression, job performance, and work productivity? J Occup Environ Med 2008;50(4):401-10.

37. Smit F, Cuijpers $P$, Oostenbrink J, et al. Costs of nine common mental disorders: implications for curative and preventive psychiatry. $J$ Ment Health Policy Econ 2006;9:193-200.

38. Lagerveld SE, Bültmann U, Franche RL, et al. Factors associated with work participation and work functioning in depressed workers: a systematic review. J Occup Rehabil 2010;20:275-92. 